\title{
Generation of Curved Planar Reformations from Magnetic Resonance Images of the Spine
}

\author{
Tomaž Vrtovec ${ }^{1,2}$, Sébastien Ourselin² ${ }^{2}$ Lavier Gomes $^{3}$, \\ Boštjan Likar ${ }^{1}$, and Franjo Pernuš ${ }^{1}$ \\ ${ }^{1}$ University of Ljubljana, Faculty of Electrical Engineering, Slovenia \\ \{tomaz.vrtovec, bostjan.likar, franjo.pernus\}@fe.uni-lj.si \\ 2 BioMedIA Lab, Autonomous Systems Laboratory, CSIRO ICT Centre, Australia \\ sebastien.ourselin@csiro.au \\ ${ }^{3}$ Department of Radiology, Westmead Hospital, University of Sydney, Australia \\ lgomes@mail .usyd.edu.au
}

\begin{abstract}
We present a novel method for curved planar reformation (CPR) of spine images obtained by magnetic resonance (MR) imaging. CPR images, created via a transformation from image-based to spinebased coordinate system, follow the structural shape of the spine and allow the whole course of the curved structure to be viewed in a single image. The spine-based coordinate system is defined on the $3 \mathrm{D}$ spine curve and on the axial vertebral rotation, both described by polynomial models. The 3D spine curve passes through the centers of vertebral bodies, and the axial vertebral rotation determines the rotation of vertebral spinous processes around the spine. The optimal polynomial parameters are found in an optimization framework, based on image analysis. The method was evaluated on $19 \mathrm{MR}$ images of the spine from 10 patients.
\end{abstract}

\section{Introduction}

Three-dimensional (3D) images of anatomical structures, obtained by computed tomography $(\mathrm{CT})$ or magnetic resonance (MR) imaging, are usually presented as two-dimensional (2D) planar cross-sections in the standard image reformation (i.e. axial, sagittal and coronal). However, planar cross-sections do not follow the curvature and orientation of curved anatomical structures (e.g. spine). To improve clinical evaluation and quantitative analysis, a more effective visualization is achieved by curved planar reformation (CPR), where cross-sections are represented in the coordinate system of the $3 \mathrm{D}$ curved structure.

Different approaches to reformation of CT spine data have already been introduced and reported to be useful in evaluating spinal deformities. Rothman et al. 1] obtained curved coronal images by connecting manually selected points into a continuous curve. After reformation, structures such as nerve roots, facet joints and spinal cord could be observed in a single image. Congenital spine abnormalities were examined by Newton et al. 2], who improved the identification of the deformities by generating curved multiplanar reformatted images of the whole spine, which were obtained by manually outlining the boundaries of the 
spine curve. Roberts et al. 3] reformatted the images orthogonal to the long axis of the neural foramina of the cervical region of the spine. By oblique reformation, they improved the consistency in the interpretation of neural foraminal stenosis between observers. In [4], the authors focused on vertebral morphology that is of significant importance in pedicle-screw placement for the treatment of scoliosis. In order to obtain true values of pedicle width, length and angle, the MR spine images were reformatted so that measurements could be performed in cross-sections perpendicular to the vertebral bodies. In contrast to the reported manual methods, an automated CPR method for 3D spine images was recently presented by Vrtovec et al. [5], who used polynomial models to describe the spinal curvature and axial vertebral rotation in the spine-based coordinate system. However, their method was developed for CT images only and is therefore modality dependent. Dedicated commercial software or software provided by $\mathrm{CT} / \mathrm{MR}$ scanner vendors allows the generation of multiplanar and curved cross-sections, however, the points that are connected into a continuous curve have to be manually selected by the user, which requires navigation through complex (spine) anatomy.

MR has become the dominant modality in imaging of the spine. Intial poor resolution of the bone structures on early MR machines was improved by the better signal-to-noise ratio (SNR), obtained from dedicated multichannel spine coils. Moreover, as there is no exposure to ionizing radiation, MR is considered to be the modality of choice for follow up examinations and longitudinal studies. However, the detection of bone structures in MR images remains a challenging task. In this paper, we present a novel method for automated CPR of MR spine images, based on the transformation from the standard image-based to the spinebased coordinate system. The transformation parameters, represented by the $3 \mathrm{D}$ spine curve and axial vertebral rotation, are obtained automatically in an optimization-based framework.

\section{Method}

\subsection{Spine-Based Coordinate System}

The standard image-based reformation is represented by the Cartesian coordinate system, where axes $x, y$ and $z$ represent the standard sagittal, coronal and axial direction, respectively. The spine-based coordinate system [5] is represented by the axes $u, v$ and $w$, which are defined on a continuous curve $c(n)$ that goes through the same anatomical landmarks on individual vertebrae and represents the $3 \mathrm{D}$ spine curve, and by the rotation $\varphi(n)$ of the vertebral spinous processes around the spine curve that represents the axial vertebral rotation (Fig. 1a). The continuous variable $n$ parameterizes the spine domain:

- The axis $w$ is tangent to the $3 \mathrm{D}$ spine curve $c(n)=(x(n), y(n), z(n))$,

- The axis $v$ is orthogonal to axis $w$ and oriented in the direction of vertebral spinous processes. The angle $\varphi(n)=\angle\left(v(n), y^{\prime}(n)\right)$ between the axis $v$ and the corresponding projection $y^{\prime}$ of the Cartesian axis $y$ onto the plane that 
is orthogonal to the $3 \mathrm{D}$ spine curve determines the rotation of the axis $v$ around the $3 \mathrm{D}$ spine curve.

- The axis $u$ is orthogonal to axis $v$ (and hence to axis $w$ ) and extends in the direction parallel to the vertebral transverse processes.

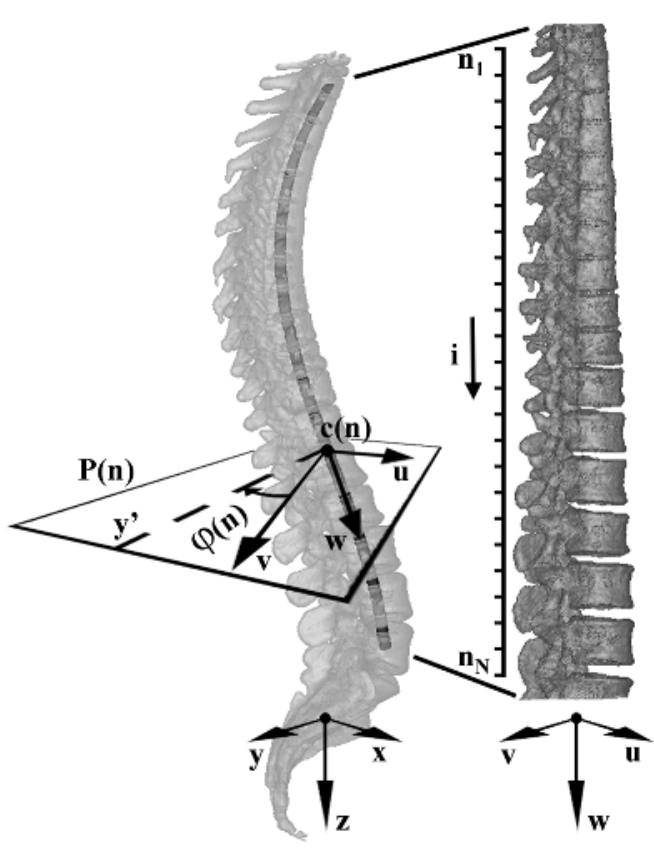

(a)

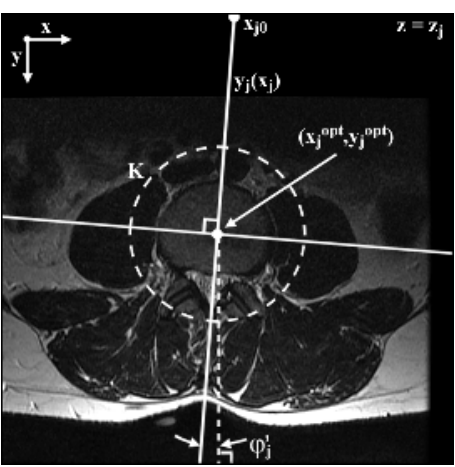

(b)

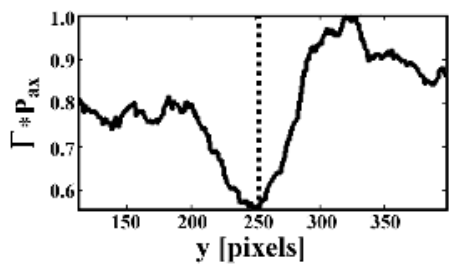

(c)

Fig. 1. (a) Transformation from the image-based to spine-based coordinate system is based on the 3D spine curve $c(n)$ and axial vertebral rotation $\varphi(n)$. (b) The center of the vertebral body in an axial cross-section is found on the line that splits the image into two mirror halves. (c) Typical response of the entropy-based operator $\Gamma$ on the straight line in (b). The minimum coincides with the center of the vertebral body.

Since the 3D spine curve and the axial vertebral rotation are expected to be smooth and continuous functions of $n$, they are parameterized by polynomial functions:

$$
c(n)=\{x, y, z\}(n)=\sum_{k=0}^{K_{\{x, y, z\}}} b_{\{x, y, z\}, k} n^{k} \quad \varphi(n)=\sum_{k=0}^{K_{\varphi}} b_{\varphi, k} n^{k},
$$

where $K_{x}, K_{y}, K_{z}$ and $K_{\varphi}$ are the degrees and $b_{x}=\left\{b_{x, k} ; k=0,1, \ldots, K_{x}\right\}$, $b_{y}=\left\{b_{y, k} ; k=0,1, \ldots, K_{y}\right\}, b_{z}=\left\{b_{z, k} ; k=0,1, \ldots, K_{z}\right\}$ and $b_{\varphi}=\left\{b_{\varphi, k}\right.$; $\left.k=0,1, \ldots, K_{\varphi}\right\}$ are the parameters of polynomials $x(n), y(n), z(n)$ and $\varphi(n)$, respectively. The parameters $b=b_{x} \cup b_{y} \cup b_{z} \cup b_{\varphi}$, specific for the spine, determine the transformation to the spine-based coodinate system and the generation of 
CPR images. For the purpose of implementation, the continuous spine domain is discretized into $N$ samples, $n_{i} ; i=1,2, \ldots, N$. The discretization yields a discrete 3D spine curve $c\left(n_{i}\right)=c\left(x\left(n_{i}\right), y\left(n_{i}\right), z\left(n_{i}\right)\right) ; i=1,2, \ldots, N$ and a discrete axial vertebral rotation $\varphi\left(n_{i}\right) ; i=1,2, \ldots, N$.

\subsection{Determination of the 3D Spine Curve}

Let the 3D spine curve be described by a curve $c(n)$ that passes through the centers of vertebral bodies, parametrized by the $3 \mathrm{D}$ spine curve parameters $b_{c}=b_{x} \cup b_{y} \cup b_{z}$. The optimal parameters $b_{c}^{o p t}$ are obtained in an optimization procedure that minimizes the sum of distances $\operatorname{dist}(\cdot)$ from the curve $c(n)$ to the centers of vertebral bodies, represented by a set of points $\{p\}$ in 3D:

$$
b_{c}^{o p t}=\underset{b_{c}}{\arg \min } \sum_{i=1}^{N} \operatorname{dist}\left(\{p\}, c\left(n_{i}\right)\right) \mid b_{c} .
$$

The centers of vertebral bodies $\{p\}$ are determined in each axial cross-section $z=z_{m} ; m=1,2, \ldots, Z$ of the 3D image. A straight line $y_{m}\left(x_{m}\right)=\tan \left(\frac{\pi}{2}-\right.$ $\left.\varphi_{m}^{\prime}\right)\left(x_{m}-x_{m 0}\right)$ that splits the vertebral body into two mirror halves and passes through the vertebral spinous process is first obtained by maximizing the similarity between the two halves (Fig. 1 b). The location $\left(x_{m}^{o p t}, y_{m}^{o p t}\right)$ of the vertebral bodies and intervertebral discs, which appear as circular areas of homogeneous intensity values, is found by minimizing the response of the entropy-based operator $\Gamma$, which consists of $K$ concentric circular rings, on the obtained straight line $y_{m}\left(x_{m}\right)$ (Fig. 11 ):

$$
\Gamma=\frac{\sum_{k=1}^{K} w_{k} H_{k}}{H \sum_{k=1}^{K} w_{k}} ; \quad w_{k}=e^{-\frac{(k-1)^{2}}{2\left(\frac{K-1}{S}\right)^{2}}} ; \quad k=1,2, \ldots, K,
$$

where $H_{k}=-\sum_{j=1}^{J} p_{j, k} \log p_{j, k}$ is the entropy of the $k^{t h} \operatorname{ring}\left(p_{j, k}\right.$ is the probability distribution of the intensity values in $k^{t h} \operatorname{ring}$ ) and $H=-\sum_{j=1}^{J} p_{j} \log p_{j}$ is the entropy of the area covered by all rings $\left(p_{j}\right.$ is the probability distribution of the intensity values). The histograms are computed using $J$ bins. The weights $w_{k}$ are chosen to be within $S$ standard deviations of the normal distribution. By repeating this procedure for all axial cross-sections, we obtain a set of points $\{p\}=\left\{x_{m}^{o p t}, y_{m}^{o p t}, z_{m}\right\} ; m=1,2, \ldots, Z$.

\subsection{Determination of the Axial Vertebral Rotation}

The angle $\varphi_{m}^{\prime} ; m=1,2, \ldots, Z$ that represents the inclination of the straight line that splits the vertebral body into two mirror halves from the sagittal reference plane $x=$ const. is determined in the standard, image-based coordinate system. As it does not take into account the sagittal and coronal vertebral inclination, it is an approximation of the true axial vertebral rotation. The true axial vertebral rotation $\varphi(n)$, parameterized by the rotational parameters $b_{\varphi}$, is obtained by 
maximizing the sum of similarities $\operatorname{sim}(\cdot)$ of the mirror halves in planes $P\left(n_{i}\right)$; $i=1,2, \ldots, N$ normal to the $3 \mathrm{D}$ spine curve and centered at the $3 \mathrm{D}$ spine curve samples $c\left(n_{i}\right) ; i=1,2, \ldots, N$, along the whole $3 \mathrm{D}$ spine curve:

$$
b_{\varphi}^{o p t}=\underset{b_{\varphi}}{\arg \max } \sum_{i=1}^{N} \operatorname{sim}\left(P\left(n_{i}, \varphi_{i}\right)\right) \mid b_{\varphi} .
$$

\subsection{Curved Planar Reformation}

CPR images are obtained by following the computed 3D spine curve $c(n)$ and axial vertebral rotation $\varphi(n)$ and by folding the obtained curved surfaces onto a plane. The curved transformation does not preserve distances, however, by applying the inverse transformation distances can be measured in the imagebased coordinate system.

\section{Experiments and Results}

\subsection{Data and Implementation Details}

The images used in this study were 18 axial spinal scans of the lumbar or thoracic spinal regions from 9 patients, acquired with a spine array coil on a $1.5 \mathrm{~T} \mathrm{MR}$ scanner (General Electric Signa Excite). For each patient, corresponding $\mathrm{T}_{1}$ weighted (average $T_{R} / T_{E}=550 / 15 \mathrm{~ms}$ ) and $\mathrm{T}_{2}$-weighted (average $T_{R} / T_{E}=$ $4560 / 110 \mathrm{~ms}$ ) images were obtained with the matrix size $X \times Y=512 \times 512$ pixels. The average voxel size was $S_{x} \times S_{y}=0.398 \times 0.398 \mathrm{~mm}^{2}$, the thickness $S_{z}=3 \div 6 \mathrm{~mm}$ and the number of axial cross-sections $Z=23 \div 31$. In addition, one whole-length $\mathrm{T}_{2}$-weighted spine image $\left(Z=208, S_{z}=3 \mathrm{~mm}\right)$ was used to test the performance of the algorithm on the whole length of the spine.

User interaction was limited to pinpointing the center of the vertebral body in only one axial cross-section, which served as the initialization for the search of the set $\{p\}$ (Eq. 2), and determination of the spinal region of the selected cross-section (cervical/thoracic/lumbar). The result was used to initialize the procedure in the subsequent cross-sections. Depending on the spinal region of the cross-section, the size of the entropy-based operator $\Gamma$ (Eq. 3) was automatically adjusted to $K=30 \div 70 \mathrm{~mm}$ (the ring width was $1 \mathrm{~mm}$ and the weights were within $S=2$ standard deviations of the normal distribution). The 3D spine curve $c(n)$ and axial vertebral rotation $\varphi(n)$ (Eq. 1) were initialized as polynomials of $1^{\text {st }}$ degree (i.e. straight lines). In a hierarchical optimization scheme (Eq. 2 and 4), the degree of polynomials was gradually increased up to $K_{\{x, y, z, \varphi\}}=5$. The number of samples $N$ was set to the number of axial crosssections. The simplex method in multidimensions was used as the optimization procedure. Standard mutual information was used as the similarity measure (function $\operatorname{sim}(\cdot))$ and $J=16$ bins were used for the computation of histograms. Euclidean distance was used as the distance measure (function $\operatorname{dist}(\cdot)$ ) 

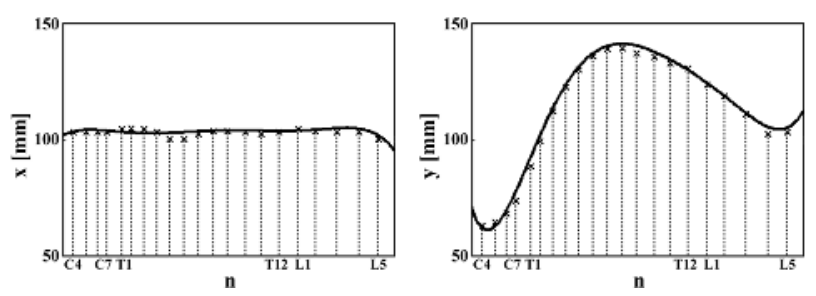

(a)

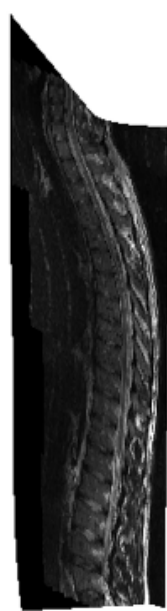

sagittal

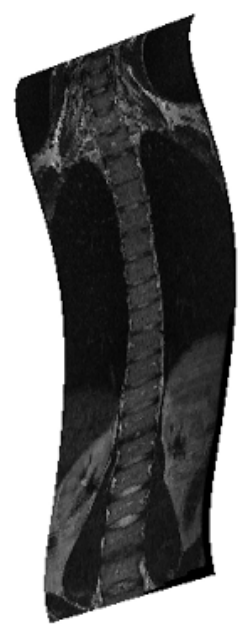

coronal

(b)

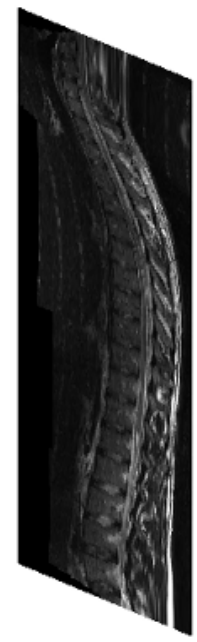

sagittal

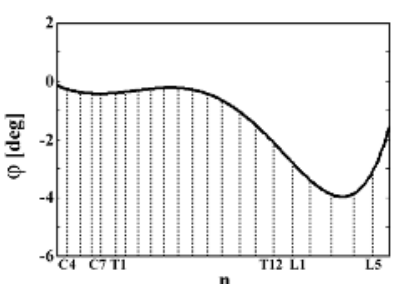

$\mathbf{n}$

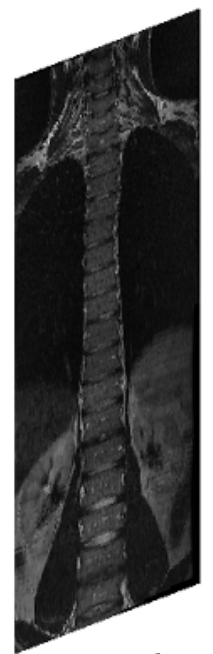

coronal

(c)

Fig. 2. (a) Course of the coronal $(x(n)$, left) and sagittal spine curve $(y(n)$, middle), showing the S-shape of the normal whole-length spine, and axial vertebral rotation $(\varphi(n)$, right), in the spine domain. The vertical lines represent the centers of vertebral bodies of ground truth data. Sagital and coronal CPR images are obtained by folding the curved surfaces (b) onto a plane (c).

In order to quantitatively assess the performance of the method, landmarks were manually placed in 3D at the centers of vertebral bodies and at the tips of vertebral spinous processes, representing ground truth data.

\subsection{Results}

By following the course of the computed 3D spine curve $c(n)$ and of the computed axial vertebral rotation $\varphi(n)$ in the spine domain $n$ (Fig. 2a), CPR images of an arbitrary view can be generated (Fig. 2b,c and 3), allowing the whole length of the spine to be observed in a single 2D image. The CPR images were successfully created from the whole-length spine image and from images of smaller spinal regions. The minimal distance between $c(n)$ and the centers of vertebral bodies in ground truth data was measured in $3 \mathrm{D}$ for all images in this study. The computed 
$\varphi(n)$ was compared to the angles obtained from ground truth data (i.e. angles between the lines through the center of vertebral bodies and the corresponding tips of vertebral spinous processes, and the sagittal reference plane). The results are presented in Table 1 and show that the method is insensitive to $\mathrm{T}_{1}$ - or $\mathrm{T}_{2}$-weighted images. We compared the courses of $c(n)$ and $\varphi(n)$ of the whole-length spine to the courses for the CT images presented in [5], and they proved to be consistent.

Table 1. Comparison between the computed 3D spine curve and axial vertebral rotation, and ground truth data (WLS denotes the whole-length spine image)

\begin{tabular}{lccccccccccc}
\hline Image number & $\mathbf{1}$ & $\mathbf{2}$ & $\mathbf{3}$ & $\mathbf{4}$ & $\mathbf{5}$ & $\mathbf{6}$ & $\mathbf{7}$ & $\mathbf{8}$ & $\mathbf{9}$ & WLS & Mean \\
\hline \hline \multicolumn{10}{c}{ Mean 3D spine curve difference $[\mathrm{mm}]$} \\
\hline $\mathrm{T}_{1}$-weighted & 2.6 & 4.3 & 1.1 & 2.5 & 2.8 & 3.4 & 2.4 & 2.1 & 2.0 & - & $\mathbf{2 . 6}$ \\
$\mathrm{T}_{2}$-weighted & 3.4 & 3.8 & 1.3 & 1.5 & 1.7 & 2.5 & 1.1 & 1.7 & 1.6 & 2.3 & $\mathbf{2 . 1}$ \\
\hline \multicolumn{10}{c}{ Mean axial vertebral rotation } & difference $[$ deg] \\
\hline $\mathrm{T}_{1}$-weighted & 4.6 & 1.2 & 1.9 & 0.7 & 2.0 & 2.0 & 0.5 & 1.7 & 1.0 & - & $\mathbf{1 . 7}$ \\
$\mathrm{T}_{2}$-weighted & 3.0 & 2.7 & 2.0 & 1.7 & 1.7 & 1.3 & 0.7 & 1.1 & 0.8 & 1.7 & $\mathbf{1 . 7}$ \\
\hline
\end{tabular}

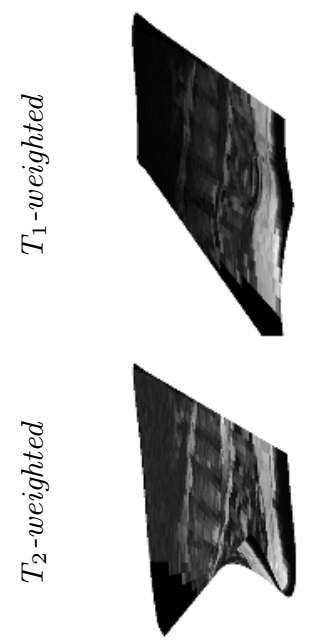

sagittal
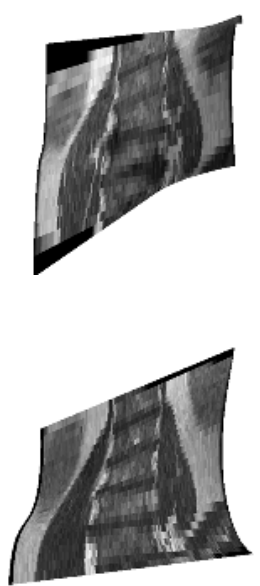

coronal

(a)
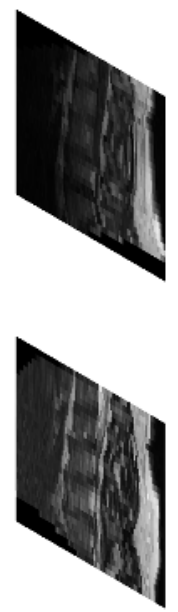

sagittal
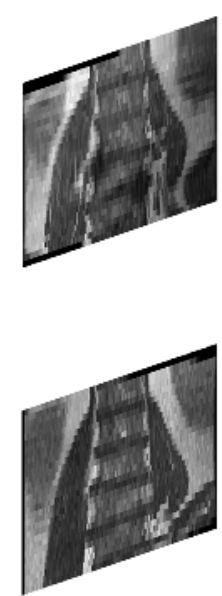

coronal

(b)

Fig. 3. CPR images, created by folding curved surfaces (a) onto a plane (b) from a $\mathrm{T}_{1}$-weighted image (top row) and a $\mathrm{T}_{2}$-weighted image (bottom row) of a spinal region

\section{Discussion}

Spinal curvature [6/7/8] and axial vertebral rotation [9,10,11/12,13/14/15] were the subject of many research studies, as they are important in surgical planning, 
analysis of surgical results and monitoring the progression of spinal deformities. Our method allows to measure both quantities automatically.

The presented method for CPR of MR spine images allows inspection and analysis of images in the coordinate system of the spine. The $3 \mathrm{D}$ spine curve and axial vertebral rotation are described continuously along the whole spinal length in a low-parametric form, namely by polynomials of up to $5^{\text {th }}$ degree. The use of CPR is valuable in clinical evaluation as it can reduce the structural complexity in favour of an improved feature perception of the spine. The representation of pathological and healthy anatomy in the same coordinate system allows an objective evaluation of pathology, especially in case of significant coronal (i.e. scoliosis) or sagittal (i.e. kyphosis or lordosis) spinal curvature. The major limitation of the presented method is that the determination of axial vertebral rotation strongly depends on the prior estimation of the $3 \mathrm{D}$ spine curve, as the rotation is measured in planes centered in the estimated spine curve samples.

Knowledge on the location and orientation of the spine in 3D can be exploited by other image analysis techniques and applied in a clinical environment, for example for the identification and measurement of dimensions of the spinal canal and the spinal cord. The notion of the spine-based coordinate system is modalityindependent and can therefore be used for data fusion, i.e. merging of CT and MR images of the same patient. We will focus our future research on these topics.

\section{References}

1. Rothman, S., Dobben, G., Rhodes, M., Glenn, W.J., Azzawi, Y.M.: Computed tomography of the spine: Curved coronal reformations from serial images. Radiology 150 (1984) 185-190

2. Newton, P., Hahn, G., Fricka, K., Wenger, D.: Utility of three-dimensional and multiplanar reformatted computed tomography for evaluation of pediatric congenital spine abnormalities. Spine 27 (2002) 844-850

3. Roberts, C., McDaniel, N., Krupinski, E., Erly, W.: Oblique reformation in cervical spine computed tomography: A new look at an old friend. Spine 28 (2003) 167-170

4. Liljenqvist, U., Allkemper, T., Hackenberg, L., Link, T., Steinbeck, J., Halm, H.: Analysis of vertebral morphology in idiopathic scoliosis with use of magnetic resonance imaging and multiplanar reconstruction. Journal of Bone and Joint Surgery 84 (2002) 359-368

5. Vrtovec, T., Likar, B., Pernuš, F.: Automated curved planar reformation of 3D spine images. Physics in Medicine and Biology 50 (2005) 4527-4540

6. Stokes, I., Bigalow, L., Moreland, M.: Three-dimensional spinal curvature in idiopathic scoliosis. Journal of Orthopaedic Research 5 (1987) 102-113

7. Perdriolle, R., Vidal, J.: Thoracic idiopathic scoliosis curve evolution and prognosis. Spine 10 (1985) 785-791

8. Drerup, B., Hierholzer, E.: Assessment of scoliotic deformity from back shape asymmetry using an improved mathematical model. Clinical Biomechanics 11 (1996) 376-383

9. Nash, C., Moe, J.: A study of vertebral rotation. Journal of Bone and Joint Surgery 51 (1969) 223-229 
10. Aaro, S., Dahlborn, M.: Estimation of vertebral rotation and the spinal and rib cage deformity in scoliosis by computer tomography. Spine 6 (1981) 460-467

11. Krismer, M., Sterzinger, W., Christian, H., Frischhut, B., Bauer, R.: Axial rotation measurement of scoliotic vertebrae by means of computed tomography scans. Spine 21 (1996) 576-581

12. Birchall, D., Hughes, D., Hindle, J., Robinson, L., Williamson, J.: Measurement of vertebral rotation in adolescent idiopathic scoliosis using three-dimensional magnetic resonance imaging. Spine 22 (1997) 2403-2407

13. Hecquet, J., Legaye, J., Duval-Beaupere, G.: Access to a three-dimensional measure of vertebral axial rotation. European Spine Journal 7 (1998) 206-211

14. Rogers, B., Haughton, V., Arfanakis, K., Meyerand, E.: Application of image registration to measurement of intervertebral rotation in the lumbar spine. Magnetic Resonance in Medicine 48 (2002) 1072-1075

15. Kuklo, T., Potter, B., Lenke, L.: Vertebral rotation and thoracic scoliosis in adolescent idiopathic scoliosis. Journal of Spinal Disorders \& Techniques 18 (2005) 139-147 